\title{
Entre prazeres e dores: um retrato da epidemia de HIV/AIDS em mulheres de 30 a 59 anos de idade
}

\author{
Between pleasures and pains: a portrait of the HIV/AIDS epidemic in women aged 30 to \\ 59 years
}
Entre placeres y dolores: un retrato de la epidemia de VIH/SIDA en mujeres de 30 a 59 años

Kátia Mariana Sousa ${ }^{1 *}$, Marília Borborema Rodrigues Cerqueira.

\section{RESUMO}

Objetivo: Analisar como a epidemia de HIV/AIDS se comporta entre mulheres de 30 a 59 anos, no estado de Minas Gerais, na região Sudeste e no Brasil. Métodos: É um estudo exploratório, transversal e descritivo. A principal fonte de dados utilizados foi o Departamento de Informática do Sistema Único de Saúde - DATASUS. A coleta de dados ocorreu entre os meses de agosto de 2019 e março de 2020 . Foram estudados os anos de 1996 (CID-10) a 2018 (último ano fechado) e foram calculadas as medidas de ocorrência, prevalência e incidência, para mulheres situadas nas faixas etárias de 30 a 59 anos. Para os dados de mortalidade, foram observados as Categorias B20 a B24. Resultados: Há elevação da incidência e prevalência de HIV/AIDS entre mulheres de 30 a 59 anos e os óbitos de mulheres, nessa mesma faixa etária, somaram $72,50 \%$ no país, $73,30 \%$ na região e $73,34 \%$ no estado de Minas Gerais, do total de mulheres vivendo com o vírus. Conclusão: Conclui-se que as mulheres deste estudo são vulneráveis ao HIV/AIDS pela falta de políticas públicas com foco nelas e, entre outras, por questões de gênero.

Palavras-chave: HIV, Síndrome de Imunodeficiência Adquirida, Mulheres.

\section{ABSTRACT}

Objective: To analyze how the HIV/AIDS epidemic is among women aged 30 to 59 years, in the state of Minas Gerais, in the Southeast and in Brazil. Methods: It is an exploratory, cross-sectional and descriptive study. The main source of data used was the Informatics Department of the Unified Health System - DATASUS. Data collection took place between 2019, August and 2020, March. The years 1996 (IDC-10) to 2018 (last closed year) were studied and the measures of occurrence, prevalence and incidence were calculated for women located in the age groups of 30 to 59 years. For mortality data, it was taking into account Categories B20 to B24. Results: There is an increase in the incidence and prevalence of HIV/AIDS among women aged 30 to 59 years and the deaths of women, in this same age group, totaled $72.50 \%$ in the country, $73.30 \%$ in the region and $73.34 \%$ in the state of Minas Gerais, of the total number of women living with the virus. Conclusion: It is concluded that the women in this study are vulnerable to HIV/AIDS due to the lack of public policies focusing on them and, among others, due to gender issues.

Keywords: HIV, Acquired Immunodeficiency Syndrome, Women.

\section{RESUMEN}

Objetivo: Analizar cómo se comporta la epidemia de VIH/SIDA en mujeres de 30 a 59 años, en el estado de Minas Gerais, en el Sureste y en Brasil. Métodos: Es un estudio exploratorio, transversal y descriptivo. La principal fuente de datos utilizada fue el Departamento de Informática del Sistema Único de Salud DATASUS. La recolección de datos se realizó entre agosto de 2019 y marzo de 2020. Se estudiaron los años 1996 (CIE-10) a 2018 (último año cerrado) y se calcularon las medidas de ocurrencia, prevalencia e incidencia para mujeres ubicadas en los grupos de edad de 30 a 59 años. Para los datos de mortalidad, se observaron las Categorías B20 a B24. Resultados: Se observa un aumento en la incidencia y prevalencia del VIH/SIDA entre mujeres de 30 a 59 años y las muertes de mujeres, en este mismo grupo de edad, totalizaron $72,50 \%$ en el país, $73,30 \%$ en la región y $73,34 \%$ en el estado de Minas Gerais, del número total de mujeres que viven con el virus. Conclusión: Se concluye que las mujeres de este estudio son vulnerables al VIH/SIDA debido a la falta de políticas públicas enfocadas en ellas y, entre otras, por cuestiones de género.

Palabras clave: VIH, Síndrome de Inmunodeficiencia Adquirida, Mujeres.

1 Universidade Estadual de Montes Claros (Unimontes), Montes Claros - MG.

*E-mail: katiasousa2215@gmail.com 


\section{INTRODUÇÃO}

As mulheres, ao longo de suas trajetórias, vivenciam profundas mudanças em seus corpos, em seus relacionamentos, podendo reconhecer-se ou não como vulneráveis às Doenças Sexualmente Transmissíveis (DST), como o vírus da imunodeficiência humana (HIV), causador da síndrome da imunodeficiência adquirida (AIDS) (MIRANDA-RIBEIRO P, et al., 2009).

A vulnerabilidade, um arcabouço teórico que consegue abraçar praticamente todas as possibilidades de estudo sobre o HIV/AIDS, tem os componentes: individual (ligado aos conhecimentos, comportamento e características ligadas à pessoa), o social (refere-se ao contexto social no qual a pessoa se insere) e o programático (que diz sobre o apoio institucional, os serviços de saúde, a disponibilidade de meios de cuidado, como a distribuição de preservativos, serviços médicos, etc.)(SÁNCHEZ AIM e BERTOLOZZI MR, 2007).

A partir desse conceito e no caso da epidemia de HIV/AIDS entre mulheres, amplia-se o olhar para o tema, pois há particularidades sobre a vida da mulher que exigem os conhecimentos de cada um dos componentes do referido arcabouço teórico para o estudo.

Para as autoras Santos NJS, et al. (2009), a relação entre homens e mulheres, no exercício da sexualidade, é marcada por uma submissão feminina e responsabilização pela contracepção e concepção, observando-se dificuldades em estabelecer diálogo entre as partes e resultando em maior vulnerabilidade das mulheres. Nesse contexto, é imprescindível pensar em prevenção tendo em vista as relações de gênero e poder existentes nessas relações. $O$ estudo das autoras revelou que as mulheres com diagnóstico positivo para o HIV/AIDS eram mais velhas, menos escolarizadas, com maior taxa de desemprego e, com menor registro de união estável, além de demonstrar que não há uma diferença estaticamente significativa entre o número de parceiros sexuais entre os dois grupos -aquelas que vivem com o HIV/AIDS e aquelas que não vivem.

Em paralelo a essas percepções, as mulheres têm apresentado índices crescentes de infecção pelo HIV. Alguns autores apontam que esse grupo é mais vulnerável biologicamente a serem infectadas por meio de relações sexuais do que os homens (BASTOS FI, 2001; KORNFIELD SVL, 2010). De acordo com Bastos FI (2001), o fator biológico é decorrente da extensão da mucosa vaginal que é exposta ao sêmen, que por sua vez, tem maior concentração do HIV que o líquido vaginal e, o contato do sêmen ejaculado se espalha pelo canal vaginal. A não identificação, por parte de algumas mulheres, com comportamentos e grupos de risco, é um fator que aumenta a sua vulnerabilidade ao vírus (VILLELA WV e BARBOSA RM, 2017).

No Brasil, de 2007 a junho de 2018 foram notificados 169.932 casos de infecção pelo HIV, destes, 31,4\% (77.812) são mulheres. Destas, $96,8 \%$ dos casos, acima de 13 anos de idade, inserem-se na categoria de exposição heterossexual e 41,9\% são brancas (BRASIL, 2018). Ou seja, o contato sexual é uma importante via de contração do vírus.

No entanto, ainda nos tempos atuais, existem tabus relacionados à sexualidade, principalmente à feminina, corroborando para que as mulheres não consigam estabelecer um diálogo com seus parceiros sobre o uso do preservativo. A dependência emocional e financeira e os medos do abandono e da solidão as tornam suscetíveis a relações desprotegidas. Essa atitude é, por muitas vezes, considerada como prova de amor e fidelidade ao parceiro (VON MUHLEN BK, et al., 2014). A rejeição ao uso da camisinha é associada aos homens, entretanto, algumas mulheres reproduzem a ideia de que é "como chupar bala com papel" e de que a pausa para colocar o preservativo "quebra o clima" - esse cenário é reproduzido no título deste artigo com a palavra "prazeres". Para essas, a camisinha é vista, sobretudo, como um método contraceptivo (MIRANDARIBEIRO P, et al., 2009).

A associação do preservativo com a contracepção suscita outro fator de vulnerabilidade feminina às doenças sexualmente transmissíveis, o climatério. De acordo com a Organização Mundial da Saúde (OMS, 1996), o climatério é definido como o processo de transição entre o período fértil e não fértil da mulher, iniciando-se entre os 35 e 40 anos e persistindo até os 65 anos. Após o fim desse período de fertilidade, a contracepção deixa de ser necessária, podendo reduzir ainda mais o uso da camisinha, ademais, as alterações ocorridas no período da pós-menopausa, no órgão vaginal, podem aumentar o risco de contrair outras DST, além do HIV/AIDS (NARASIMHAN M, et al., 2016). 
As relações afetivo-sexuais estáveis, como o casamento, são vistas como um elo protetor contra as doenças. Um fato que merece destaque é que mesmo as mulheres que se enxergam como vulneráveis dentro de um relacionamento não fazem uso de medidas preventivas; nessa perspectiva, a vulnerabilidade e as políticas preventivas perpassam por caminhos diversos e complexos (MIRANDA-RIBEIRO P, et al., 2009; COSTA FM, et al., 2014).

Villela WV e Barbosa RM (2017) dizem que, apesar da tendência de feminização da epidemia no Brasil, as mulheres não são o grupo prioritário das políticas de prevenção, visto que parte dos diagnósticos ocorre no período gestacional, para o qual existem as políticas de saúde materno-infantil. As autoras citam, ainda, que os contextos em que ocorre a infecção são distintos, mas apresentam fatores em comum, como as histórias de amor, a falta de conhecimento sobre as DST de forma geral e sobre as formas de contaminação e, até mesmo, a pressão dos parceiros.

Nesse sentido, os achados de Mesenburg MA, et al. (2017) indicam que a prevalência de realização do teste para diagnóstico do HIV/AIDS é mais comum durante a realização do pré-natal, se comparado à realização do teste por demanda espontânea, sendo ainda maior para mulheres que já tiveram pelo menos uma gestação; logo, existe uma parcela da população feminina que por não ter filhos biológicos ou por não ter tido assistência pré-natal, nunca realizou o teste. Embora haja o processo de feminização da epidemia, algumas mulheres ainda se veem invulneráveis ao HIV/AIDS.

Os estudos apontam que o HIV/AIDS ainda é vista como uma doença do outro e a contaminação do outro é justificada por comportamentos considerados promíscuos. Para Zarei N, et al. (2017), o estigma existente no contexto da epidemia de HIV/AIDS, o estigma percebido pelas mulheres que vivem com o vírus é associado com o decréscimo na qualidade de vida delas - e esse fato é referendado no título como "dores", como também em alusão ao momento vivido pós-diagnóstico.

Os autores Figueiredo LG, et al. (2013) ressaltam que todos os indivíduos são e estão vulneráveis à infecção pelo vírus HIV e essa vulnerabilidade está aliada a fatores socioculturais, existência de gargalos nas políticas públicas, de conscientização e assistência. Há que se considerar a vulnerabilidade de gênero que, conforme Lima M e Schraiber LB (2013), diz respeito aos contextos de vida das mulheres, quando os componentes afetivos e as situações de violência contribuem para as dificuldades no cuidado com a saúde delas. Nesse sentido, Cerqueira MBR, et al. (2020) usam a expressão 'vulnerabilidades e desigualdades de gênero', em referência aos diversos pontos já expostos.

Assim, o objetivo deste trabalho é analisar como a epidemia se comporta entre mulheres de 30 a 59 anos, no estado de Minas Gerais, na região Sudeste e no país, visto que esse grupo não se enquadra como prioritário nas políticas de prevenção ao HIV/AIDS adotadas no país.

\section{MÉTODOS}

O estudo tem caráter exploratório, transversal e descritivo. A principal fonte de dados utilizados foi o Departamento de Informática do Sistema Único de Saúde (DATASUS). A coleta de dados ocorreu entre os meses de agosto de 2019 e março de 2020.

Para fins deste estudo, são utilizados dados referentes ao estado de Minas Gerais, à região Sudeste e ao Brasil, considerando o número de casos diagnosticados por local de residência, sexo, faixa etária e escolaridade; estimativas populacionais; internações e mortalidade associadas à síndrome da imunodeficiência humana. As variáveis 'categoria de exposição hierárquica' e 'raça/cor' foram excluídas por apresentarem um percentual de ignorados acima de $10 \%$ do total, fundamentando-se em orientações de Chackiel J (1986) para dados faltosos.

Foram estudados os anos de 1996 (Classificação Internacional de Doenças - CID na 10a . revisão) a 2018 (último ano fechado disponível). Para esses anos, foram calculadas as medidas de ocorrência, prevalência e incidência, para mulheres situadas nas faixas etárias de 30 a 59 anos. Para os dados de mortalidade, foram computadas as Categorias B20, B21, B22, B23 e B24 do CID-10. A faixa etária definida busca preencher a lacuna em termos de trabalhos publicados e, igualmente, trazer à tona a questão do aumento de casos de HIV/AIDS entre mulheres em fase de climatério. 


\section{RESULTADOS E DISCUSSÃO}

No período de 1996 a 2018, foram diagnosticados no Brasil 845.599 casos de HIV/AIDS no Brasil, desses, 410.854 são de pessoas que residem no Sudeste; essa, por sua vez, de acordo com as estimativas populacionais do Instituto Brasileiro de Geografia e Estatística (IBGE) para 2018, é também a região com maior contingente de habitantes do país, cerca de 87,7 milhões, ou $42,07 \%$ da população brasileira (DATASUS, 2020). Em Minas Gerais, verificou-se, no mesmo período um registro de 61.004 diagnósticos de HIV/AIDS. Em relação ao crescimento no número de diagnósticos efetuados, em pessoas do sexo feminino, nas regiões analisadas, foram encontrados dois resultados diferentes. O acréscimo foi de $39,13 \%$ e $60,05 \%$ entre o primeiro ano e o último em estudo, para Minas Gerais e o Brasil, respectivamente.

Contrapondo esse cenário, a região Sudeste apresentou um decrescimento de 19,60\% para o grupo de mulheres e período em estudo. Fazem-se necessários novos estudos, abordando os outros estados da região Sudeste. Os resultados apresentados acompanham a tendência mundial de heterossexualização e feminização da epidemia (VALADARES ALR, et al., 2010), trazendo para o debate as questões de gênero e de transmissão do vírus pela via sexual (Gráfico 1).

Gráfico 1 - Razão de sexos para o estado de Minas Gerais, região Sudeste e Brasil.

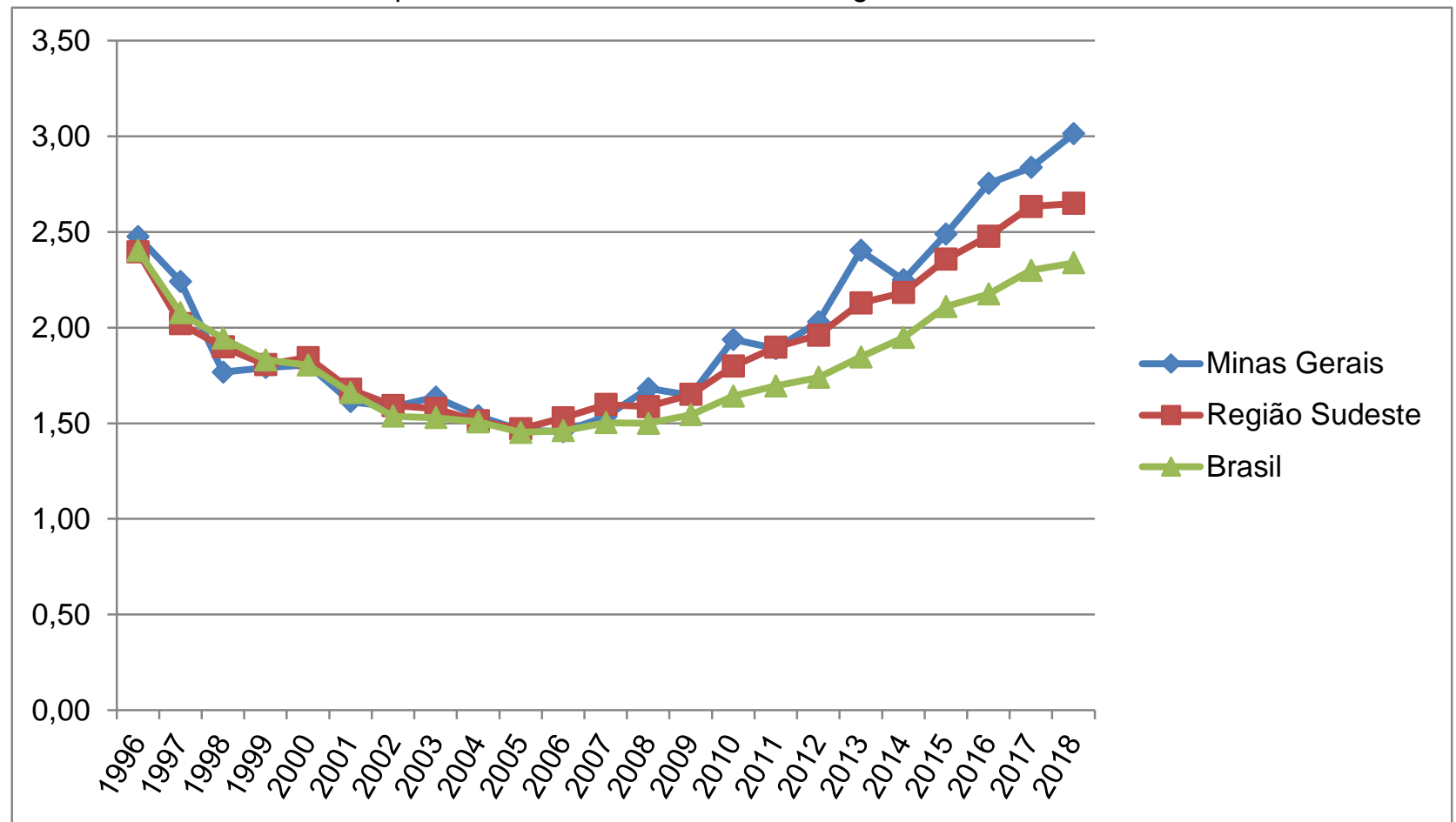

Fonte: Sousa KM e Cerqueira MBR, 2020, dados extraídos do DATASUS, 2019.

No que se refere às incidências de HIV/AIDS, tanto em Minas Gerais quanto no Brasil, houve elevação para as mulheres entre 35 a 59 anos de idade. Os dados mostram que na região Sudeste, a ocorrência de novos casos expandiu nos dois últimos intervalos estudados - 50a 54 anos e 55 a 59 anos (Tabela 1). Esses números podem ser associados à dificuldade encontrada por esse grupo na negociação do uso do preservativo, como já exposto anteriormente, que é, por muitas vezes, considerado um tabu e dispensável nas relações afetivas estáveis (LOURENÇO GO, et al., 2018).

O climatério, à medida que promove alterações fisiológicas e efeitos na autoestima da mulher, fragilizandoa, aumenta a suscetibilidade a relações sexuais menos seguras e por consequência aumenta a vulnerabilidade a DST (VALADARES ALR, et al., 2010). A Tabela 1 revela, ainda, que a prevalência é elevada em todos os intervalos quinquenais e regiões. Os resultados encontrados estão associados ao aumento da incidência e também ao sucesso da terapia antirretroviral, respondendo por uma maior sobrevivência de pessoas que vivem com o HIV/AIDS. 
Tabela 1 - Incidência e prevalência do HIV/AIDS a cada 10 mil mulheres por faixa etária, para Minas Gerais, região Sudeste e Brasil, anos 1996 e 2018.

\begin{tabular}{|c|c|c|c|c|c|c|c|c|c|c|c|c|}
\hline \multirow[b]{3}{*}{ Faixa etária/Ano } & \multicolumn{4}{|c|}{ Minas Gerais } & \multicolumn{4}{|c|}{ Sudeste } & \multicolumn{4}{|c|}{ Brasil } \\
\hline & \multicolumn{2}{|c|}{ Incidência } & \multicolumn{2}{|c|}{ Prevalência } & \multicolumn{2}{|c|}{ Incidência } & \multicolumn{2}{|c|}{ Prevalência } & \multicolumn{2}{|c|}{ Incidência } & \multicolumn{2}{|c|}{ Prevalência } \\
\hline & 1996 & 2018 & 1996 & 2018 & 1996 & 2018 & 1996 & 2018 & 1996 & 2018 & 1996 & 2018 \\
\hline 30 a 34 anos & 1,38 & 0,96 & 18,87 & 53,55 & 3,56 & 1,39 & 62,62 & 119,25 & 2,31 & 1,74 & 38,05 & 87,07 \\
\hline 35 a 39 anos & 0,89 & 1,00 & 15,94 & 47,41 & 2,62 & 1,67 & 50,02 & 99,10 & 1,81 & 2,04 & 31,08 & 75,09 \\
\hline 40 a 44 anos & 0,72 & 1,22 & 11,12 & 42,36 & 1,93 & 1,59 & 36,39 & 81,58 & 1,29 & 2,05 & 22,92 & 64,92 \\
\hline 45 a 49 anos & 0,42 & 1,16 & 8,27 & 35,61 & 1,59 & 1,56 & 26,49 & 62,50 & 0,99 & 1,89 & 15,96 & 50,90 \\
\hline 50 a 54 anos & 0,31 & 0,85 & 6,26 & 24,82 & 1,04 & 1,42 & 18,22 & 42,55 & 0,67 & 1,61 & 11,09 & 35,96 \\
\hline 55 a 59 anos & 0,32 & 0,87 & 3,80 & 18,05 & 0,68 & 0,97 & 12,46 & 27,74 & 0,43 & 1,27 & 7,59 & 24,64 \\
\hline
\end{tabular}

Fonte: Sousa KM e Cerqueira MBR, 2020, dados extraídos do DATASUS, 2019.

A maior sobrevivência de pessoas que vivem com o vírus e, neste artigo, o caso das mulheres de 30 a 59 anos, revela o protagonismo do Brasil ao adotar um modelo de resposta ao HIV/AIDS com a oferta de tratamento 100\% pelo SUS, desde 1996 (GRECO DB, 2016). Contudo, o aumento das incidências é o resultado, também, da redução no volume de recursos disponíveis para os trabalhos de prevenção e tratamento do HIV/AIDS, desde 2011, e tem contribuído para o retrocesso no controle do HIV/AIDS no país, como um todo (ENSP, 2015).

Nos três, Minas Gerais, região Sudeste e Brasil, quando os dados são filtrados considerando o nível educacional do grupo das mulheres de 30 a 59 anos de idade, é obtido o mesmo padrão: as que se declaram ter, da quinta a oitava série incompleta, representam cerca de $30 \%$ dos diagnósticos segundo escolaridade $(30,16 \%, 30,57 \%$ e $31,03 \%$ para Minas Gerais, Sudeste e Brasil, respectivamente); ocorre ainda um decrescimento dos novos casos em analfabetas e com primeira a quarta série incompleta de $54,84 \%, 89,35 \%$ e $62,71 \%$ para Minas Gerais, Sudeste e Brasil, nessa ordem.

As mulheres da faixa etária em estudo e com curso superior incompleto e completo perfazem, em média, 3,78\% para a série em estudo, no país; na região Sudeste, 3,73\%; no estado de Minas Gerais, 2,92\% do total de mulheres cuja escolaridade foi registrada nos formulários de notificação compulsória. Os dados conhecidos sobre escolaridade das mulheres em pauta representam aproximados $36,11 \%$ do total dos casos de HIV/AIDS dessas mulheres, no Brasil; e 32,98\% e 32,31\% em, respectivamente, região Sudeste e Minas Gerais. Aqui, ressalta-se a premente necessidade de melhoria nos registros compulsórios, oferecendo elementos para as pesquisas e para subsidiar a elaboração de políticas públicas.

Nada obstante, os achados sobre a escolaridade das mulheres da análise corroboram com aqueles encontrados por Zarei N, et al. (2017), por Villela WV e Barbosa RM (2017) e por Pinho AA, et al. (2017), no que se refere ao perfil das mulheres que vivem com HIV/AIDS, ou seja, expresso pelo baixo nível de escolaridade e revelando um quadro de vulnerabilidade social. Villela WV e Barbosa RM (2017) afirmam que esse foi o perfil das mulheres vivendo com o vírus no país ao longo dos anos de epidemia, caracterizandose, portanto, como um indicador das políticas anti-HIV voltadas para um grupo específico de mulheres, as gestantes, uma vez que as demais, em geral, não são o grupo prioritário das políticas de prevenção.

Entre 1998 e 2018, foram registradas no Brasil 755.970 internações por doenças associadas ao HIV; dessas, 36,02\% foram para pacientes do sexo feminino. Quando observado por grupos etários, percebeu-se que $66,53 \%$ da morbidade das mulheres pertencem à faixa etária em estudo e, entre essas, o maior número foi para o intervalo de 35 a 39 anos (45.570 internações).

Chama a atenção a parcela de mulheres de 30 a 59 anos internadas em relação às internações totais de mulheres, em decorrência do HIV/AIDS, nos espaços geográficos em estudo, perfazendo valores acima de $50 \%$ durante todo o período, alcançando picos de 74,04\% em 2015, 74,76\% em 2016 e 81,92\% em 2017, nessa ordem, Brasil, região Sudeste e Minas Gerais (Gráfico 2). 
A vida das mulheres que vivem com HIV/AIDS é pautada pelo estigma existente no contexto da epidemia, como também, pelo estigma internalizado, impedindo-as de cuidarem de si mesmas, de terem completa adesão à terapia antirretroviral e, portanto, resultando em possíveis internações. Zarei N, et al. (2017) discutem sobre esses eventos de saúde, ressaltando que aquelas mulheres que vivem com HIV/AIDS e não têm sintomas físicos relacionados ao HIV têm melhor qualidade de vida. Já Quatremère G, et al. (2017) abordam a questão dos efeitos colaterais da terapia antirretroviral nas mulheres que vivem com o vírus, citando o efeito negativo no estado de saúde autorreferido e concluindo que mudanças no regime da terapia são medidas comuns adotadas por essas mulheres.

Gráfico 2 - Proporção (\%) de internações de mulheres de 30 a 59 anos, pelo SUS, em relação ao total de mulheres internadas em decorrência do HIV/AIDS, Brasil, região Sudeste e Minas Gerais, 1998-2018.

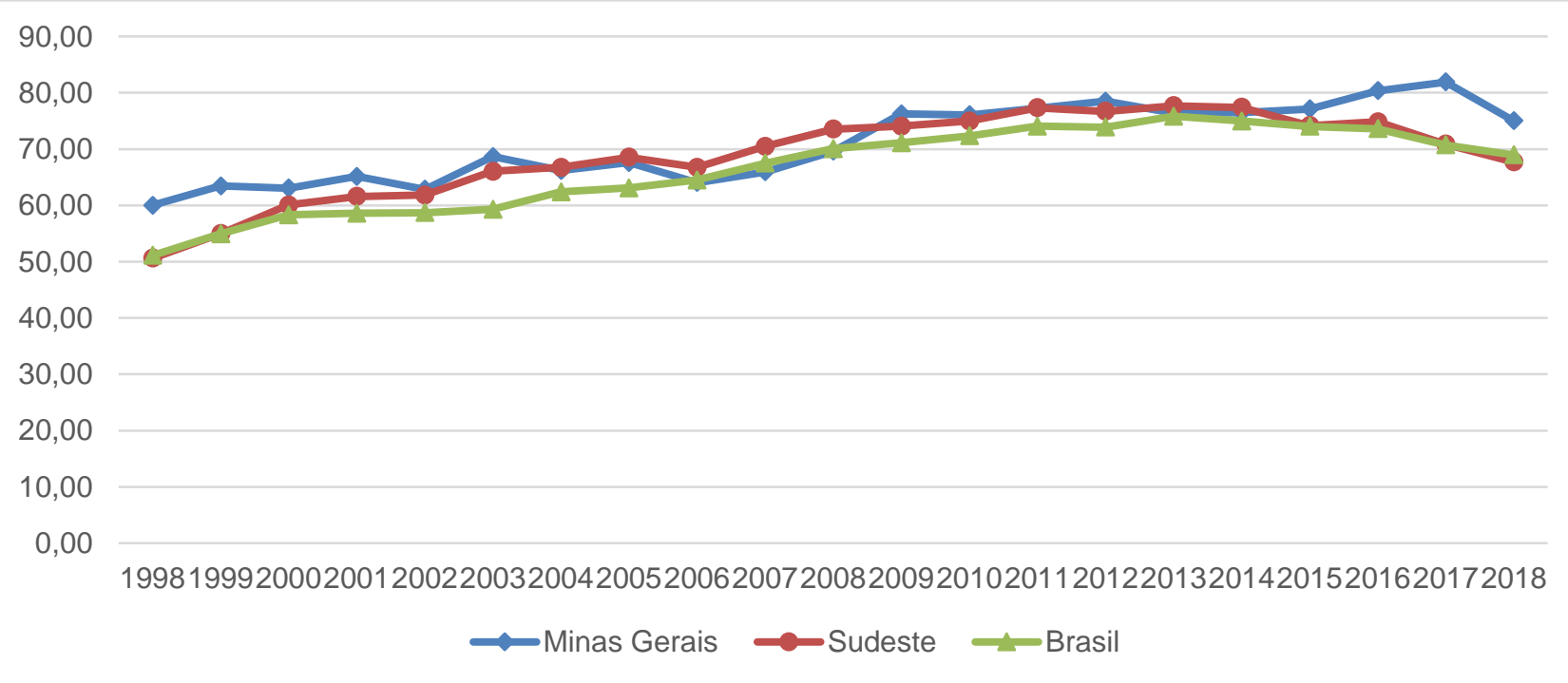

Fonte: Sousa KM e Cerqueira MBR, 2020, dados extraídos do DATASUS, 2019.

No Brasil, ocorreram 259.369 óbitos por HIV/AIDS, no período estudado;138.903 foram no Sudeste e, em Minas Gerais, 18.423 óbitos. Do total de óbitos do país, 32,12\% foram mulheres, assim como na região Sudeste e em Minas Gerais, foram observados percentuais iguais 32,04\% e 31,82\%, em ordem. Entre os óbitos de mulheres, aqueles na faixa etária de estudo (30 a 59 anos), somaram 72,50\% no país, 73,30\% na região e 73,34\% no estado de Minas Gerais. Desagregando esses dados de óbitos por faixa etária, os resultados mostraram que nas três regiões, os registros de óbitos de pessoas do sexo feminino foram maiores entre 30 a 44 anos- óbitos prematuros, representando, respectivamente, para o Brasil, Sudeste e Minas Gerais, $48,66 \%, 48,60 \%$ e $46,46 \%$ do total de mortes entre mulheres.

Nesse ponto, retoma-se o tema da direcionalidade das políticas públicas de prevenção e combate ao HIV/AIDS, considerando que a mortalidade decorrente da síndrome (AIDS) pode ser um desfecho do retardo no diagnóstico devido à dificuldade de acesso aos serviços de saúde e informações, como também mencionado por Lopes $\mathrm{F}$ (2003) e Braga $\mathrm{P}$, et al. (2007). Outro tema que vem à baila é a desigualdade de gênero, recaindo a responsabilidade de cuidado da família, dos filhos e das tarefas domésticas sobre a mulher quando ela poderia estar atenta às mudanças do seu próprio corpo (PEREIRA ECA, et al., 2008).

Sobre os resultados, reforça-se a necessidade de melhoria nos registros, considerando-se o percentual de dados ignorados quando se analisa por anos de estudo da paciente, como encontrado também por Reis AC, et al. (2007). No ano em observação, no país, o maior número de óbitos foi registrado no intervalo etário de 30 a 44, são 481 óbitos de pessoas do sexo feminino por HIV/AIDS, e essas, em termos de anos de estudo, registraram de 4 a 7 anos e, somadas as outras categorias que refletem a escolaridade, o número de óbitos atinge 1.536; a região Sudeste e o estado de Minas Gerais reproduzem o mesmo padrão, perfazendo 177 e 23 registros de óbitos para mulheres com 4 a 7 anos de estudo, em sequência, como encontrado em outro estudo(PEREIRA ECA, et al., 2008) (Tabela 2). 
Tabela 2 - Número de óbitos de mulheres por doenças relacionadas ao HIV/AIDS, segundo faixa etária e anos de estudo, Brasil, região Sudeste e Minas Gerais, 2018.

\begin{tabular}{|c|c|c|c|c|c|c|c|}
\hline \multicolumn{8}{|c|}{ Brasil } \\
\hline Faixa Etária & Nenhuma & 1 a 3 anos & 4 a 7 anos & 8 a 11 anos & 12 anos e mais & Ignorado & Total \\
\hline 0 a 14 anos & 0 & 4 & 3 & 0 & 0 & 25 & 32 \\
\hline 15 a 29 anos & 10 & 65 & 151 & 120 & 17 & 62 & 425 \\
\hline 30 a 44 anos & 57 & 261 & 481 & 389 & 55 & 293 & 1536 \\
\hline 45 a 59 anos & 89 & 253 & 333 & 247 & 52 & 239 & 1213 \\
\hline 60 anos ou mais & 73 & 114 & 123 & 67 & 17 & 76 & 470 \\
\hline Total & 229 & 697 & 1091 & 823 & 141 & 695 & 3666 \\
\hline \multicolumn{8}{|c|}{ Região Sudeste } \\
\hline Faixa Etária & Nenhuma & 1 a 3 anos & 4 a 7 anos & 8 a 11 anos & 12 anos e mais & Ignorado & Total \\
\hline 0 a 14 anos & 0 & 0 & 3 & 0 & 0 & 7 & 10 \\
\hline 15 a 29 anos & 1 & 24 & 55 & 52 & 8 & 18 & 158 \\
\hline 30 a 44 anos & 8 & 89 & 177 & 151 & 27 & 117 & 569 \\
\hline 45 a 59 anos & 32 & 124 & 152 & 113 & 24 & 113 & 558 \\
\hline 60 anos ou mais & 23 & 50 & 56 & 33 & 7 & 40 & 209 \\
\hline Total & 64 & 287 & 443 & 349 & 66 & 295 & 1504 \\
\hline \multicolumn{8}{|c|}{ Minas Gerais } \\
\hline Faixa Etária & Nenhuma & 1 a 3 anos & 4 a 7 anos & 8 a 11 anos & 12 anos e mais & Ignorado & Total \\
\hline 0 a 14 anos & 0 & 0 & 0 & 0 & 0 & 2 & 2 \\
\hline 15 a 29 anos & 0 & 0 & 4 & 12 & 1 & 1 & 18 \\
\hline 30 a 44 anos & 0 & 16 & 23 & 20 & 2 & 26 & 87 \\
\hline 45 a 59 anos & 7 & 19 & 17 & 17 & 2 & 25 & 87 \\
\hline 60 anos ou mais & 2 & 12 & 6 & 4 & 1 & 9 & 34 \\
\hline Total & 9 & 47 & 50 & 53 & 6 & 63 & 228 \\
\hline
\end{tabular}

Fonte: Sousa KM e Cerqueira MBR, 2020, dados extraídos do DATASUS, 2019.

O número de óbitos em decorrência do HIV/AIDS, maior nas faixas etárias de 30 a 44 anos e 45 a 49 anos suscita outras questões importantes, como a possibilidade da mortalidade nesses intervalos impactar 0 número de filhos órfãos maternos e sobre o indicador de anos de vida perdidos (REIS AC, et al., 2007), pois são mortes prematuras, nos primeiros estágios da vida.

A respeito, Traebert J, et al. (2015) ressaltam que o número de anos de vida perdidos por morte prematura, por HIV/AIDS, pode ser reduzido se os indivíduos - no contexto deste trabalho, as mulheres - iniciarem o tratamento assim que o diagnóstico for dado e, para isso, o acesso aos serviços de saúde necessita ser aprimorado. Ademais, a mortalidade por essas causas e faixas etárias foi também identificada no trabalho de Pereira ECA, et al. (2008), quando os autores sugerem o diagnóstico precoce e o tratamento adequado, como também a oferta apropriada, tendo como foco essas mulheres.

Outros autores relacionam a infecção pelo HIV/AIDS em mulheres à presença de câncer de colo uterino, pois as mulheres que vivem com o vírus são mais propensas à contaminação pelo Human Papilomavirus (HPV) do que as demais e, como consequência, mais predispostas a desenvolverem lesões uterinas (ABRAHAM AG, et al., 2013).Em conformidade com essa discussão, Gonçalves GC, et al. (2020) pontuam que, além de serviços de saúde de qualidade, são indispensáveis políticas públicas que ampliem o conhecimento sobre as relações sexuais, empoderamento e a prevenção de DST.

\section{CONCLUSÃO}

No que tange à epidemia de HIV/AIDS entre mulheres de 30 a 59 anos, no estado de Minas Gerais, na região Sudeste e no país, há elevação da incidência e prevalência de casos entre essas mulheres, de forma geral. Quanto à mortalidade de mulheres por doenças relacionadas ao HIV/AIDS, foi maior nas faixas etárias de 30 a 49 anos, concluindo-se que é necessário que essas mulheres sejam vistas como sexualmente ativas e protagonistas de suas vidas, o que resultará na indispensável reformulação e direcionamento de políticas públicas para elas. Este artigo enriquece a área de estudo por trazer um grupo de mulheres de idade específica, reforçando que todos os esforços são imprescindíveis na luta contra o HIV/AIDS e, de igual valor, contra as desigualdades e vulnerabilidades, inclusive a de gênero. 


\section{REFERÊNCIAS}

1. ABRAHAM AG, et al. Invasive cervical cancer is among HIV-infected women: a North American multicohort collaboration prospective study. J Acquir Immune Defic Syndr, 2013; 62(4): 405-413.

2. BASTOS FI. A feminização da epidemia de aids no Brasil: determinantes estruturais e alternativas de enfrentamento. Rio de Janeiro: Associação Brasileira Interdisciplinar de Aids; 2001.

3. BRASIL. Ministério da Saúde (MS). Boletim epidemiológico: HIV/AIDS. Brasília: MS; 2018.

4. CERQUEIRA MBR, et al. Vulnerabilidades e desigualdades de gênero: HIVIAIDS também é assunto de mulher. In.: DIOTTO N, et al. (org.). Estudos de gênero e feminismos na sociedade contemporânea: diálogos interdisciplinares. Cruz Alta: llustração, 2020; 2: 257-273.

5. CHACKIEL J. Studies of causes of death in Latin América current situation and future perspectives. Siena, Italy: International Union for the Scientific Study of Population; Institute of Statistics University of Siena; 1986.

6. COSTA FM, et. al. A percepção feminina quanto à vulnerabilidade de se contrair DST/AIDS. Revista da Universidade Vale do Rio Verde, Três Corações, 2014; 12(2): 879-889.

7. DATASUS. População residente por Região e Sexo, 2018.

8. FIGUEIREDO LG, et al. Percepção de mulheres casadas sobre o risco de infecção pelo HIV e o comportamento preventivo. Revista de Enfermagem. Rio de Janeiro: UERJ, 2013; 21(6): 805-811.

9. GONÇALVES GC, et al. Prevalence of women diagnosed with HIV/AIDS in Brazil: a retrospective study from 2010 to 2019. Research, Society and Development, 2020; 9(9), e96996749.

10. GRECO DB. Trinta anos de enfrentamento à epidemia da Aids no Brasil, 1985-2015. Ciência e Saúde Coletiva, 2016; 21(5): 1553-1564.

11. KORNFIELD SVL. Maternal-Fetal Attachment, Temporal Orientation and Locus of Control: Implications for Prenatal Care Behaviors and HIV Risk Reduction during Pregnancy. Tese (Doutorado em Filosofia) -Drexel University, Philadelphia, USA, 2010: $115 \mathrm{p}$.

12. LIMA M, SCHRAIBER LB. Violência e outras vulnerabilidades de gênero em mulheres vivendo com HIV/AIDS. Temas em Psicologia, 2013; 21(3): 947-960.

13. LOURENÇO GO, et al. Nem santa, nem puta, apenas mulher: a feminização do HIV/aids e a experiência de soropositividade. Sex., Salud Soc. Rio de Janeiro, 2018; 30: 262-281.

14. MESENBURG MA, et al. Teste solicitado e espontâneo: um estudo de base populacional com mulheres de uma cidade do Sul do Brasil. Cad. Saúde Pública, 2017; 33(10): e00074415.

15. MIRANDA-RIBEIRO $\mathrm{P}$, et al. É igual chupar bala com papel: a vulnerabilidade feminina ao HIV/aids e o uso de camisinha em Belo Horizonte e Recife. In: MIRANDA-RIBEIRO P, SIMÃO AB. (Org.). Qualificando os números: estudos sobre saúde sexual e reprodutiva no Brasil. 2. ed. Belo Horizonte: ABEP; UNFPA, 2009; 391-416.

16. NARASIMHAN M, et al. Ageing and healthy sexuality among women living with HIV. Reproductive Health Matters, 2016; 24(48), 43-51.

17. OMS - Organização Mundial de Saúde. Investigação sobre a menopausa nos anos noventa. Genebra, 1996.

18. PEREIRA ECA, et al. Tendência da incidência e da mortalidade por aids em mulheres na transição menopausal e pós-menopausa no Brasil, 1996-2005. Rev. Assoc. Med. Bras. São Paulo, 2008; 54(5).

19. PINHO AA, et al. Diferenças e similaridades entre mulheres que vivem e não vivem com HIV: aportes do estudo GENIH para a atenção à saúde sexual e reprodutiva. Cad. Saúde Pública, 2017; 33(12): e00057916.

20. QUATREMĖRE G, et al. How are women living with HIV in France coping with their perceived side effects of antiretroviral therapy? Results from the eve study. Plos One.2017.

21. REIS AC, et al. A mortalidade por aids no Brasil: um estudo exploratório de sua evolução temporal. Epidemiologia e Serviços de Saúde. Brasília, 2007; 16(3).

22. SÁNCHEZ AIM, BERTOLOZZI MR. Pode o conceito de vulnerabilidade apoiar a construção do conhecimento em Saúde Coletiva? Ciência \& Saúde Coletiva, 2007; 12(2): 319-324.

23. SANTOS NJS, et al. Contextos de vulnerabilidade para o HIV entre mulheres brasileiras. Cad. Sáude Pública, Rio de Janeiro, 2009, 25(2), 321-333.

24. TRAEBERT J, et al. Estimativa da carga de doença por aids em Florianópolis, Santa Catarina, Brasil, 2009. Epidemiologia e Serviços de Saúde, 2015: 24(3).

25. VALADARES ALR, et al. HIV em mulheres de meia-idade: fatores associados. Rev. Assoc. Med Bras., 2010; 56(1): 112-115.

26. VILLELA WV, BARBOSA RM. Trajetórias de mulheres vivendo com HIV/AIDS no Brasil: Avanços e permanências da resposta à epidemia. Ciência \& Saúde Coletiva, Rio de Janeiro,2017; 22(1): 87-96.

27. VON MUHLEN BK, et al. Mulheres e o HIV/AIDS: Intersecções entre gênero, feminismo, psicologia e saúde pública. Revista Colombiana de Psicología, Bogotá,2014; 23(2): 285-296.

28. ZAREI N, et al. Perceived Stigma and Quality of Life Among Women Living with HIVIAIDS. Women's Health Bull. 2017; 4(1):e34535. 